BULLETIN OF THE

AMERICAN MATHEMATICAL SOCIETY

Volume 80, Number 5, September 1974

\title{
GENERALIZED GRADIENT FIELDS AND ELECTRICAL CIRCUITS ${ }^{1}$
}

\author{
BY ALCIDES LINS NETO
}

Communicated by Jacob Feldman, January 9, 1974

1. Introduction. In On the mathematical foundations of electrical circuit theory, Smale [S.1] proposes the following two problems.

Problem 1.1. What can one say about the dynamical systems which are gradient systems with respect to a nondegenerate indefinite metric, say on a compact manifold.

Problem 1.2. Can one always regularize the equations of (1.6) [S.1], by adding arbitrarily small inductors and capacitors to the circuit appropriately? How? By regularizing we mean obtaining new equations which have the property $\pi: \sum \rightarrow \mathscr{L} \times \mathscr{C}^{\prime}$ is a local diffeomorphism.

Furthermore he makes the following conjecture

ConjeCture 1.3. Suppose $X=\operatorname{grad}(\omega)$ is the gradient of a closed 1form with respect to a Riemannian metric on a compact manifold $M$. Suppose further that $\omega$ is not cohomologous to zero and that $X$ is well behaved in the sense that it satisfies the conditions of [S.2, (2.2)]. Then $X$ has a closed orbit, not a point, which is aymptotically stable (i.e. a sink).

In this work we give a counterexample to this conjecture. Furthermore we reformulate it, solving the new version in the case $\operatorname{dim} M=2$. For Problem 1.1 we obtain generic properties for the generalized gradient fields as in the Kupka-Smale theorem. Moreover we characterize structural stability for these types of vector fields in the case $M$ is compact, orientable, and $\operatorname{dim}=2$. For Problem 1.2 we give a counterexample in the general case and solve the problem imposing conditions on the resistors of the circuit.

Before we state the theorems we need some definitions and notations. $M$ will be a $C^{\infty}$ manifold (with or without boundary), $T M \oplus T M=$ $\left\{(p, v, w) \mid p \in M, v, w \in T M_{p}\right\}$.

Definition 1.4. A metric $C^{r}$ on $M$ is a $C^{r}$ map $\mu: T M \oplus T M \rightarrow R$, such that for each $p \in M$, the map $\mu_{p}: T M_{p} \times T M_{p} \rightarrow \boldsymbol{R}$ given by $\mu_{p}(v, w)=$ $\mu(p, v, w)$ is bilinear symmetric. We say that $\mu$ is a nondegenerate metric on $M$ if for each $p \in M, \mu_{p}$ is a nondegenerate bilinear form on $T M_{p}$.

AMS (MOS) subject classifications (1970). Primary 34D30; Secondary 94D20.

${ }^{1}$ This is a resume of my dissertation, developed at IMPA under the guidance of Professor Cesar Camacho. 
Notations $1.5 . \quad \Lambda^{1, r}(M)$ is the set of $C^{r}, 1$-forms on $M$ and $\mathfrak{X}^{r}(M)$ is the set of $C^{r}$ vector fields on $M$. Here we will consider $\Lambda^{1, r}(M)$ and $\mathfrak{X}^{r}(M)$ endowed with the Whitney $C^{r}$ topology. $\mathscr{F}^{r}(M)$ will denote the set of closed $C^{r} 1$-forms on $M$ and $\xi^{r}(M)$ the set of exact $C^{r} 1$-forms on $M$.

We remark that $\xi^{r}(M) \subset \mathscr{F}^{r}(M)$ are closed linear subspaces of $\Lambda^{1, r}(M)$.

DEFINITION 1.6. Let $\mu$ be a nondegenerate metric on $M$ and $\omega \in \Lambda^{1, r}(M)$. We say that $X \in \mathfrak{X}^{r}(M)$ is the gradient of $\omega$ with respect to $\mu$ if for each $p \in M$ and $v \in T M_{p}$ we have

$$
\mu_{p}(X(p), v)=\omega_{p}(v) .
$$

In this case we denote $X=\operatorname{grad}_{\mu}(\omega)$. It is not difficult to show that the equality (1.6.1) defines a unique $C^{r}$ vector field on $M$ and that the map $\operatorname{grad}_{\mu}: \Lambda^{1, r}(M) \rightarrow \mathfrak{X}^{r}(M)$ is an isomorphism of topological vector spaces.

Notations 1.7. In this section we fix a nondegenerate metric $\mu$ on $M$.

1.7.1. $\mathscr{F}_{\mu}^{r}(M)=\operatorname{grad}_{\mu}\left(\mathscr{F}^{r}(M)\right)$ is the set of vector fields $X \in \mathfrak{X}^{r}(M)$, such that $X=\operatorname{grad}_{\mu}(\omega), \omega \in \mathscr{F}^{r}(M)$.

1.7.2. $\xi_{\mu}^{r}(M)=\operatorname{grad}_{\mu}\left(\xi^{r}(M)\right)$.

1.7.3. Let $M-S\left(\mathfrak{X}^{r}(M)\right)$ be the set of Morse-Smale vector fields on $M$ (cf. [P.3]). If $\mathscr{N} \subset \mathfrak{X}^{r}(M)$, then $\mathscr{N} \cap M-S\left(\mathfrak{X}^{r}(M)\right)$ is denoted by $M-S\left(\mathscr{A}^{\prime}\right)$.

1.7.4. Let $\mathscr{G}_{123}\left(\mathfrak{X}^{r}(M)\right)$ be the set of Kupka-Smale vector fields on $M$ (cf. [P.1]). If $\mathscr{N} \subset \mathfrak{X}^{r}(M)$, then $\mathscr{N} \cap \mathscr{G}_{123}\left(\mathfrak{X}^{r}(M)\right)$ is denoted by $\mathscr{G}_{123}(\mathscr{N})$.

1.7.5. We say that a closed $C^{1}$ curve $\gamma:[a, b] \rightarrow M$ is transversal to $\omega \in \mathscr{F}^{r}(M)$ if for each $t \in[a, b], \omega_{\gamma(t)}\left(\gamma^{\prime}(t)\right) \neq 0$.

Now we can state the results concerning Conjecture 1.3 and Problems $1.1,1.2$.

Proposition 1.8. Let $\omega \in \mathscr{F}^{r}(M)$. Then there exists a Riemannian metric $\mu$, such that $\operatorname{grad}_{\mu}(\omega)$ has a closed orbit which is an attractor if and only if $\omega$ admits a closed transversal.

THEOREM A. Let $M$ be a $C^{\infty}$ compact, orientable, 2-dimensional manifold, $\partial M=\varnothing$ and $M \neq S^{2}$. Then the set of closed 1-forms which admit a closed transversal is open and dense in $\mathscr{F}^{r}(M)$.

SKETCH OF THE PROOF. We note that only density offers some difficulty. The idea of the proof is to show that $\omega \in \mathscr{F}^{r}\left(M^{2}\right)$ can be approached by a 1 -form $\tilde{\omega} \in \mathscr{F}^{r}(M)$, such that there is a leaf $\gamma$ of the foliation induced by $\tilde{\omega}$ on $M^{2}$ which is nontrivially recurrent. Thus $\tilde{\omega}$ admits a closed transversal. We note that the perturbation of $\omega$ cannot be made locally, and in fact it is made in a tubular neighborhood of a closed curve on $M^{2}$ which intersects some leaf of $\omega$ transversally in a unique point. 
We note that it is not difficult to construct an open set of closed 1-forms on $S^{2} \times S^{1}$ which do not admit a closed transversal (note that $\xi^{r}\left(S^{2} \times S^{1}\right) \neq$ $\left.\mathscr{F}^{r}\left(S^{2} \times S^{1}\right)\right)$.

A natural question is: For which manifolds $M$ is the set of closed 1forms which admit a closed transversal dense? We conjecture that a sufficient condition for $\operatorname{dim} M \geqq 3$ is $H_{1}(M ; R) \neq 0$ and $\pi_{n-1}(M)=0$.

For Problem 1.1 we have the following results:

THEOREM B. Let $M$ be a $C^{\infty}$ manifold. Then $\mathscr{G}_{123}\left(\mathscr{F}_{\mu}^{r}(M)\right)\left[\mathscr{G}_{123}\left(\xi_{\mu}^{r}(M)\right)\right]$ is residual in $\mathscr{F}_{\mu}^{r}(M)\left[\xi_{\mu}^{r}(M)\right](\mu$ is a fixed nondegenerate metric on $M)$.

SKETCH OF THE PROOF. The idea is to use a technique introduced by Abraham in [A-R, $\S \S 31,32,33]$. We note that such techniques cannot be applied crudely to the problem when we are restricted to $\mathscr{F}_{\mu}^{r}(M)$. The following example shows the main difficulty.

Let $Q=\left\{(t, x) \in \boldsymbol{R}^{2}|(t) \leqq 1| x \mid, \leqq 1\right\}$ and $\mu$ be the metric on $\boldsymbol{R}^{2}$ whose quadratic form is $2 d t d x$. Given $f: \boldsymbol{R}^{2} \rightarrow \boldsymbol{R}$ we have $\operatorname{grad}_{\mu}(d f)=(\partial f / \partial y$, $\partial f / \partial x)$. Consider the Banach space

$$
N=\left\{\eta \in \xi^{r}\left(\boldsymbol{R}^{2}\right) \mid \eta(p)=0 \text { in } p \notin Q\right\} .
$$

Let $f: \boldsymbol{R}^{2} \rightarrow \boldsymbol{R}$ be given by $f(t, x)=x$ and $\omega=d f$. Then $\operatorname{grad}_{\mu}(\omega)=(1,0)$. Let $N_{1}$ be the open set of $N$, defined by

$$
N_{1}=\left\{\eta \in N \mid \operatorname{grad}_{\mu}(\omega+\eta)=\left(Y_{1}, Y_{2}\right) \text { with } Y_{1}(p)>\frac{1}{2} \forall p \in Q\right\} .
$$

Let $\Sigma_{1}=\{(t, x) \in Q \mid t=1\}$ and $F: N_{1} \rightarrow \Sigma_{1}$ be defined by $F(\eta)=$ the point where the orbit of $\operatorname{grad}_{\mu}(\omega+\eta)$, by the point $(-1,0)$, intersects $\Sigma_{1}$. Then $F$ is $C^{\mathbf{1}}$. To use Abraham's techniques it is essential that $F$ be a submersion. In this example $F$ is not a submersion at $\eta=0$.

This difficulty is overcome by restricting the analysis to an open and dense subset $\tau$ of $\mathscr{F}_{\mu}^{r}(M)$. Then we show that $\mathscr{G}_{123}\left(\mathscr{F}_{\mu}^{r}(M)\right)$ is residual in $\tau$.

TheOREM C. Let $M$ be a $C^{\infty}$, compact manifold with $\partial M=\varnothing$. Let $\mu$ be a Riemannian metric on $M$. Then $M-S\left(\xi_{\mu}^{r}(M)\right)$ is dense in $\xi_{\mu}^{r}(M)$.

Since the Morse-Smale vector fields are structurally stable (cf. [P-S]), it follows from Theorem $\mathrm{C}$ and minor arguments that the set of structurally stable vector fields in $\xi_{\mu}^{r}(M)$ is $M-S\left(\xi_{\mu}^{r}(M)\right)$.

Smale [S.3] proves a weaker form of Theorem C. There he perturbs both the metric $\mu$ and the 1 -form $\omega=d f$.

THEOREM D. Let $M$ be a $C^{\infty}$ compact, orientable, 2-dimensional manifold with $\partial M=\varnothing$. Let $\mu$ be a nondegenerate metric on $M$. Then $M-S\left(\mathscr{F}_{\mu}^{r}(M)\right)\left[M-S\left(\xi_{\mu}^{r}(M)\right)\right]$ is dense in $\mathscr{F}_{\mu}^{r}(M)\left[\xi_{\mu}^{r}(M)\right]$. 
The idea of the proof is to use the techniques developed by Peixoto in [P.2].

In $\S 2$ we give an example which shows that the answer to Problem 1.2, in the general case, is no. However we have the following result:

THEOREM E. Let $G$ be a circuit which satisfies:

(a) The projection $i_{L} \times v_{C}: K \rightarrow \mathscr{L} \times \mathscr{C}^{\prime}$ (cf. [S.1, p. 4]) is surjective.

(b) If $\rho$ is a resistor of $G$ and $\Lambda_{\rho}$ its characteristic curve, then $\Lambda_{\rho}$ is the graph of a function (A) $i_{\rho}=f\left(v_{\rho}\right)$ or $(\mathrm{B}) v_{\rho}=f\left(i_{\rho}\right)$, where $f: \boldsymbol{R} \rightarrow \boldsymbol{R}$ is a smooth function.

Then $G$ is regularizable in the sense of [S.1, (3.3)]. Furthermore if $G_{1}$ is the new circuit and $\pi_{1}: \Sigma_{1} \rightarrow \mathscr{L}_{1} \times \mathscr{C}_{1}^{\prime}$, then $\pi_{1}$ is a $C^{1}$ diffeomorphism.

The idea of the proof is to insert inductors in series with some of the resistors of type $A$ and capacitors in parallel with some of the resistors of type B.

\section{Examples.}

EXAMPLE 2.1. This is a counterexample to Conjecture 1.3. Let $M=$ $T^{n}=\boldsymbol{R}^{n} /(2 \pi \boldsymbol{Z})^{n}$. We have the natural covering map $\boldsymbol{R}^{n} \rightarrow^{p} T^{n}$, such that $p$ identifies points $\left(x_{1}, \cdots, x_{n}\right),\left(x_{1}^{\prime}, \cdots, x_{n}^{\prime}\right) \in \boldsymbol{R}^{n}$ where $\left(x_{i}-x_{i}^{\prime}\right) / 2 \pi \in \boldsymbol{Z}$. Let $\mu$ be the Riemannian metric on $T^{n}$ induced by the euclidean metric $\tilde{\mu}$ of $\boldsymbol{R}^{n}$. Let

$$
\tilde{\omega}\left(x_{1}, \cdots, x_{n}\right)=\sum_{i=1}^{n}\left(1-2 \sin x_{i}\right) d x_{i} .
$$

Then $\tilde{\omega}$ is closed and there exists a closed 1-form $\omega$ on $T^{n}$ such that $\tilde{\omega}=p^{*}(\omega)$. It is not difficult to see that $\omega$ is not exact. Let $X=\operatorname{grad}_{\mu}(\omega)$. Then $X$ is $C^{\infty}$ and $X=\left(X_{1}, \cdots, X_{n}\right)$ (in coordinates) where $X_{i}$ is a MorseSmale vector field on $S^{1}$ with two singularities, a sink and a source. Therefore $X$ is a Morse-Smale vector field on $T^{n}$ without closed orbits.

EXAMPLE 2.2. Let $\rho$ be a resistance such that its characteristic curve $\Lambda_{\rho} \subset R^{2}$ has tangents in all directions.

ASSERTION. Let $G$ be a circuit such that $\rho$ is the unique resistor of $G$. Then $G$ is not regular. (We suppose obviously that $\rho$ is not the unique element of $G$.) Write the currents and voltages of $G$ as

$$
(i, v)=\left(x, y, z, x^{\prime}, y^{\prime}, z^{\prime}\right),
$$

where $\left(x, x^{\prime}\right) \in \mathscr{L} \times \mathscr{L}^{\prime},\left(y, y^{\prime}\right) \in \mathscr{C} \times \mathscr{C}^{\prime}$ and $\left(z, z^{\prime}\right) \in \mathscr{R} \times \mathscr{R}^{\prime} \cong \boldsymbol{R}^{2}$ (for the notations see [S.1]). Let $\pi^{\prime}: K \rightarrow \mathscr{R} \times \mathscr{R}^{\prime}, \Sigma \subset K$ and $\pi: \Sigma \rightarrow \mathscr{L} \times \mathscr{C}^{\prime}$ be as in [S.1]. Then $\pi^{\prime}$ is surjective (because Kirchhoff laws do not impose restrictions in $\left.\mathscr{R} \times \mathscr{R}^{\prime}\right)$ and $\Sigma=\left(\pi^{\prime}\right)^{-1}\left(\Lambda_{\rho}\right)$ is a submanifold of $K$. If $p=$ $\left(x, y, z, x^{\prime}, y^{\prime}, z^{\prime}\right) \in \Sigma$ we have $T \Sigma_{p}=\left\{\left(\dot{x}, \dot{y}, \dot{z}, \dot{x}^{\prime}, \dot{y}^{\prime}, \dot{z}^{\prime}\right)=\dot{p} \mid \dot{p} \in K\right.$ and 
$\left.\left(\dot{z}, \dot{z}^{\prime}\right) \in\left(T \Lambda_{\rho}\right)_{\left(z, z^{\prime}\right)}\right\}$ and $D \pi_{p}(\dot{p})=\left(\dot{x}, \dot{y}^{\prime}\right) \in \mathscr{L} \times \mathscr{C}^{\prime}$. If there exists $p \in \Sigma$, such that $D \pi_{p}$ is surjective, then the projection $i_{L} \times v_{c}: K \rightarrow \mathscr{L} \times \mathscr{C}^{\prime}$ is surjective. Now

$$
\operatorname{dim}\left(\operatorname{ker}\left(i_{L} \times v_{c}\right)\right)=\operatorname{dim}(K)-\operatorname{dim}\left(\mathscr{L} \times \mathscr{C}^{\prime}\right)=1 .
$$

Let $\dot{p}=\left(\dot{x}, \dot{y}, \dot{z}, \dot{x}^{\prime}, \dot{y}^{\prime}, \dot{z}^{\prime}\right) \in \operatorname{ker}\left(i_{L} \times v_{c}\right), \dot{p} \neq 0$. By the hypothesis, there exists $\left(z, z^{\prime}\right) \in \Lambda_{\rho}$, such that $\left(\dot{z}, \dot{z}^{\prime}\right) \in\left(T \Lambda_{\rho}\right)_{\left(z, z^{\prime}\right)}$, therefore $\dot{p} \in T \Sigma_{p}$ where $\pi^{\prime}(p)=\left(z, z^{\prime}\right)$ and $D \pi_{p}(\dot{p})=0, \dot{p} \neq 0$. This shows that $\pi$ is not a local diffeomorphism at $p$.

\section{REFERENCES}

[A-R] Ralph Abraham, Joel Robbin and A. Kelley, Transversal mappings and flows, Benjamin, New York, 1967. MR 39 \#2181.

[P.1] M. M. Peixoto, On an approximation theorem of Kupka and Smale, J. Differential Equations 3 (1967), 214-227, MR 35 \#499.

[P.2] — Structural stability on two-dimensional manifolds, Topology 1 (1962), 101-120. MR 26 \#426.

[P.3] J. Palis, On Morse-Smale dynamical systems, Topology 8 (1968), 385-404. MR 39 \#7620.

[P-S] J. Palis and S. Smale, Structural stability theorems, Proc. Sympos. Pure Math., vol. 14 Amer. Math. Soc., Providence, R.I., 1970, pp. 223-231. MR 42 \#2505.

[S.1] S. Smale, On the mathematical foundations of electrical circuit theory, J. Differential Geometry 7 (1972).

[S.2] — Differentiable dynamical systems, Bull. Amer. Math. Soc. 73 (1967), 747-817. MR 37 \#3598.

[S.3] —, On gradient dynamical systems, Ann. of Math. (2) 74 (1961), 199-206. MR 24 \#A2973.

Instituto de Matemática Pura e Aplicada, Rio de Janeiro, Brasil 